\title{
Der geraubte Räuber
}

\section{Fanfiction aus der Romanfabrik um 1800 (Vulpius, Brückner)}

\section{Erika Thomalla}

Angenommen: 20. Oktober 2021 / Online publiziert: 22. November 2021

(C) Der/die Autor(en) 2021

Zusammenfassung Der Beitrag befasst sich am Beispiel von Christian August Vulpius' Roman Rinaldo Rinaldini mit frühen Formen moderner Fanfiction. Die Fortsetzungen des Romans von fremder Hand arbeiten in unterschiedlicher Weise daran, jene Probleme, die im Original ungelöst bleiben, zu beheben und der kontingenten Ereignisfolge ein Telos zu verleihen. Das Beispiel zeigt, dass Fanfiction die unrealisierten Möglichkeiten literarischer Werke nutzt, indem sie durch eigenwillige Lektüren die Zwangsläufigkeit von Handlungsverläufen hinterfragt und alternative Welterklärungsmodelle entwirft. Zugleich lässt sich an Vulpius' Roman beobachten, inwiefern Vorlage und Fanfiction hier in einem Wechselverhältnis stehen. Die Untersuchung von Fanfiction eröffnet damit auch Perspektiven für eine netzwerkförmige Literaturgeschichtsschreibung.

Erika Thomalla $(\bowtie)$

Institut für deutsche Literatur, Humboldt-Universität zu Berlin, Unter den Linden 6, 10099 Berlin, Deutschland

E-Mail: erika.thomalla@hu-berlin.de 


\section{The robbed Robber}

Fanfiction from the Novel Factory around 1800 (Vulpius, Brückner)

Abstract Using Christian August Vulpius' novel Rinaldo Rinaldini as an example, this article deals with early forms of modern fanfiction. The unauthorized sequels to the novel explore different ways to remedy problems that remain unsolved in the original version and to find a telos for the contingent sequence of events. The example shows that fanfiction makes use of the unrealized possibilities of literary works by questioning the inevitability of plot developments through idiosyncratic readings and designing alternative models for explaining the world. At the same time, Vulpius' novel demonstrates the extent to which the original novel and the works of fanfiction are interrelated. The study of fanfiction thus also opens up perspectives for a network-based historiography of literature.

Manchmal erweist es sich als vorteilhaft, keinen Plan zu haben. Der Weimarer Hofbibliothekar und Dramaturg Christian August Vulpius, der mit seinem Räuberroman Rinaldo Rinaldini 1799 einen überraschenden Publikumserfolg erzielte, stand bei der zeitgenössischen Literaturkritik beständig im Verdacht, seine Werke aus »schaalen Einfällen « willkürlich und »bunt « zusammenzustellen. ${ }^{1}$ Vulpius, so der Tenor der Rezensenten, sei ein »Possenreisser«, der beim Schreiben kaum je einen »verständigen Plan« verfolge: »Alles geht drunter und drüber, ohne Zweck und Ziel, ohne Einheit und Zusammenhang. $\ll^{2}$ Seine »Manier« zu erzählen sei »die abgeschmackteste die sich ersinnen läßt «. ${ }^{3}$ Selbst wohlwollende Rezensenten bemerkten, dass die »Abentheuer « bei Vulpius »zu sehr gehäuft« würden »und eben dadurch unwahrscheinlich « erschienen. ${ }^{4}$ Dabei sei der Räuberromanautor Vulpius, der seine »Abentheuer nach Willkühr « zusammenflicke, selbst ein Räuber: »Eigentlich ist der Verfasser von einerlei Handwerk mit seinem Helden [...]. Er hat für sein Buch alles zusammen gestohlen, was in irgend einem andern neuen Romane Glück gemacht hatte. $\aleph^{5}$

Der Vorwurf der planlosen Zusammenstellung schematischer Motive und Erzählelemente zielt nicht zuletzt darauf, jene Texte, die sich als ein Phänomen der neuesten Mode präsentieren, als unoriginelle Neuauflagen eines veralteten Genres auszuweisen. Vulpius griff nicht nur im Rinaldo Rinaldini vorzugsweise auf eine episodische Erzählform zurück, die er aus dem barocken Schelmen- und Abenteuerroman über-

\footnotetext{
1 Adolph Freiherr von Knigge, »Die Männer der Republik; Sie konnts nicht übers Herz bringen«, in: Ders., Rezensionen 1779-1797 in Friedrich Nicolais »Allgemeine deutsche Bibliothek und »Neue allgemeine deutsche Bibliothek«, hrsg. Ernst August Freiherr Knigge, Göttingen 2009, 238.

2 Anonym, »Rinaldo Rinaldini, ein Schauspiel in fünf Aufzügen, vom Verfasser des Romans gleiches Namens«, in: Neue allgemeine deutsche Bibliothek, 58. Band (1801), 364-365.

3 Anonym, »Fürstinnen unglücklich durch Liebe«, in: Neue allgemeine deutsche Bibliothek, 72. Band (1802), 357-358.

4 Anonym, »Rinaldo Rinaldini, der Räuberhauptmann«, in: Neue allgemeine deutsche Bibliothek, 51. Band (1800), 35-36, hier: 36.

5 Garlieb Helwig Merkel, »Briefe an ein Frauenzimmer über die wichtigsten Produkte der schönen Literatur«, Zweiter Band, Berlin 1801, 415 und 419.
} 
nommen hatte. ${ }^{6}$ Damit widersetzte er sich den Ansprüchen der Romantheorie des späten 18. Jahrhunderts. ${ }^{7}$ Nach der Auffassung Friedrich von Blanckenburgs, der die neuen Paradigmen des Erzählens 1774 in seinem Versuch über den Roman ausformulierte, sollten die Autoren von Romanen eine »kleine Welt« kreieren, in der Figuren durch die zweckmäßige Verbindung aller Ereignisse eine psychologische Entwicklung durchleben und allmählich zur »Vervollkommnung « hingeführt werden. ${ }^{8}$ Vulpius' Held Rinaldini erlebt demgegenüber weder eine sinnhafte Welt, noch macht er eine Entwicklung zur Perfektibilität durch. Er verstrickt sich über drei Bände, die mehrfach fortgesetzt wurden, immer wieder in Abenteuer, lernt neue Frauen und neue Orte kennen, muss wiederholt fliehen, träumt von einem ruhigen Leben und kommt nie ans Ziel.

Diese serielle Erzählweise, die nicht zuletzt dadurch begünstigt wurde, dass Vulpius auf schriftstellerische Nebenverdienste angewiesen war und aufgrund der Bezahlung nach Druckbögen »Masse produzieren « musste, ${ }^{9}$ erschien allerdings nur einem kleinen Teil der Leserinnen und Leser unzeitgemäß oder geschmacklos. Beim breiten Publikum erwiesen sich gerade das scheinbar veraltete Romanmodell und das damit verbundene Fehlen großer erzählerischer Bögen als Erfolgsrezepte. Die offene Struktur des Romans bot die Möglichkeit, der Geschichte immer wieder neue, unvorhergesehene Wendungen zu verleihen und die Spannung dadurch über mehrere Jahre aufrechtzuerhalten. Darüber hinaus bestand der Reiz der Erzählung darin, dass der vormoderne narrative Bau von einem Protagonisten belebt wurde, der Züge eines modernen, empfindsamen Charakters trägt: Vulpius' Räuberhauptmann ist nicht bloß ein affektgetriebener, abenteuerfreudiger Gesetzloser, sondern auch und vor allem ein »melancholischer « Held, der durch sein Leiden an einer scheinbar vom Chaos bestimmten Romanwelt die Empathie der Leserschaft weckte. ${ }^{10}$ Mehrfach erwägt Rinaldini den Suizid, weil ihm die Rückkehr in ein geregeltes Leben ebenso unmöglich erscheint wie die Fortführung seiner Räuberexistenz. Zusätzlich verkomplizierend kommt hinzu, dass es im Roman einige Indizien dafür gibt, dass das, was als zufälliger Ereignisverlauf erscheint, eigentlich durch die Interessen geheimer Mächte gelenkt wird. Dieses Geheimbundmotiv wird wie viele andere Erzählstränge immer wieder aufgegriffen und weiterentwickelt, ohne dass sich je restlos aufklärt, wer oder was dahintersteht.

\footnotetext{
6 Dass Vulpius bereits durch seine frühen Lektüreerfahrungen und seine Tätigkeit als Mitarbeiter der »Bibliothek der Romane « die Form des episodischen Reihenromans als wesentliches Gattungsvorbild entdeckte, referiert Andreas Meier, »Die >triviale Klassik< - Unterhaltungsliteratur als kulturelles Komplement«, in: Christian August Vulpius. Eine Korrespondenz zur Kulturgeschichte der Goethezeit, hrsg. Andreas Meier, Berlin, New York 2003, XL-CXCII, hier: XCV.

7 Vgl. Wilhelm Vosskamp, Romantheorie in Deutschland. Von Martin Opitz bis Friedrich von Blanckenburg, Stuttgart 1973, $142 \mathrm{ff}$.

8 Friedrich von Blanckenburg, Versuch über den Roman (1774), Stuttgart 1965, 166.

9 Ute Schneider, »Literatur zur Unterhaltung der Massen: Christian August Vulpius auf dem Buchmarkt um 1800«, in: Alexander Košenina (Hrsg.), Andere Klassik. Das Werk von Christian August Vulpius, 2. durchgesehene Auflage, Hannover 2013, 26-38, hier: 29.

10 Roberto Simanowski, Die Verwaltung des Abenteuers. Massenkultur um 1800 am Beispiel Christian August Vulpius, Göttingen 1988, 338; vgl. auch Mark-Georg Dehrmann, »Rinaldo Rinaldini der Räuberhauptmann«, in: Košenina (Hrsg.) (Anm. 9), 143-147, hier: 146.
} 
Der Roman entwirft damit in mehrfacher Hinsicht ein Spannungsfeld zwischen Offenheit und Geschlossenheit bzw. zwischen Zufall und Zweckbestimmtheit. ${ }^{11}$ Während die narrative Struktur das Geschehen kontingent und unabgeschlossen erscheinen lässt, wird auf der Inhaltsebene durch die Figuren fortwährend die Suche nach Sinnhaftigkeit und Zusammenhang betrieben, ohne dass die Handlung je an einen Ziel- oder Endpunkt käme. Der Infragestellung einer »metaphysisch-providentiell garantierten Realität « durch einen »kontingenten Erzählprozess « ${ }^{12}$ wird eine Vielzahl an möglichen teleologischen Zielpunkten entgegengesetzt, die sich allesamt als nicht tragfähig erweisen. Dieses Spannungsverhältnis ließ Rinaldo Rinaldini nicht nur über Jahrzehnte zum begehrtesten Buch in den Leihbibliotheken werden, ${ }^{13}$ sondern ist zugleich der Grund dafür, dass der Roman mehrfach von fremder Hand fortgesetzt oder umgeschrieben wurde. Das Schicksal des Räuberhauptmanns, der sich einer »eindimensionalen Interpretation « ebenso widersetzte wie der Einordnung in ein »stimmiges Figurenkonzept $«,{ }^{14}$ stieß bei den Leserinnen und Lesern auf so großes Interesse, dass sie sich mit den Fortsetzungen des Autors nicht zufriedengeben oder sie teilweise noch nicht einmal abwarten wollten, sondern seine Abenteuer eigenmächtig aneigneten. Diese Fortsetzungen von fremder Hand können als Vorläufer oder frühe Beispiele jener produktiven Rezeptionsformen literarischer Texte gelten, die etwa seit Mitte des 20. Jahrhunderts als Fanfiction bezeichnet werden. ${ }^{15}$

\section{I.}

Unter dem Begriff Fanfiction werden Texte verstanden, die bereits existierende fiktionale Charaktere weiterentwickeln, indem sie ihre Geschichte fortschreiben, ihnen ein anderes Ende verleihen, sie von Neben- zu Hauptfiguren machen oder ihre Vorgeschichten erzählen. ${ }^{16}$ Fanfiction unterscheidet sich damit von anderen Adaptionsformen literarischer Texte, die lediglich Plotstrukturen oder einzelne Motive aus ihren Vorlagen übernehmen. In der Gegenwart entsteht Fanfiction überwiegend in international vernetzten Fangemeinschaften, in denen Amateure meist ohne kommerzielles Interesse alternative Handlungsverläufe oder Enden ihrer favorisierten Bücher und Serien verfassen und sich darüber austauschen. Auch wenn man diese moderne Fankultur mit den Produktions- und Rezeptionsbedingungen literarischer Texte um 1800 nur bedingt vergleichen kann, gibt es doch Kontinuitäten, die es sinnvoll erscheinen lassen, die Anfänge der modernen Fanfiction im späten 18. Jahr-

11 Zu diesem Spannungsfeld, das zahlreiche Romane im späten 18. Jahrhundert auszeichnet, siehe Werner Frick, Providenz und Kontingenz. Untersuchungen zur Schicksalssemantik im deutschen und europäischen Roman des 17. und 18. Jahrhunderts, 2 Bde., Tübingen 1988.

12 Ebd., 12.

13 Vgl. Dehrmann (Anm. 10), 144.

14 Vgl. Meier (Anm. 6), CXIX.

15 Zur Geschichte des Begriffs, der ursprünglich verwendet wurde, um Texte über Fans zu bezeichnen, siehe Karen Hellekson und Kristina Busse, »Introduction«, in: Dies. (Hrsg.), The Fan Fiction Studies Reader, Iowa City 2014, 1-17, hier: $5 \mathrm{f}$.

$16 \mathrm{Vgl}$. Aaron Schwabach, Fan Fiction and Copyright. Outsider Works and Intellectual Property Protection, London, New York 2011, 8. 
hundert anzusiedeln. In diesem Zeitraum nehmen Publikationen, die das Schicksal eines literarischen Charakters weiterverfolgen, Romanen ein alternatives Ende verleihen oder Vor- bzw. Nebenhandlungen auserzählen, stark zu. ${ }^{17}$

Die Gründe für diese Entwicklung sind vielfältig: Sie liegen unter anderem in der Umstellung auf den Konditionshandel, der für die Buchhändler ein höheres finanzielles Risiko bedeutete und die Werbung mit erfolgreichen Geschichten und Charakteren attraktiv machte, sowie in fehlenden Regelungen zum Urheberrecht an fiktiven Charakteren, die vor der Aneignung durch fremde Autorinnen und Autoren nicht geschützt waren. ${ }^{18}$ Vor allem aber verdankt sich die Herausbildung von Fanfiction als eines breiten, populären Phänomens einer in der zweiten Hälfte des 18. Jahrhunderts entstandenen Lektürepraxis, die auf »identifikatorischer, in kompetenter Form sogar empathischer Teilhabe an den fiktionalen Charakteren « beruht. ${ }^{19}$ Das impliziert nicht nur den Vorsatz, die vermeintlich aus dem Text hervorgehenden Einstellungen, Gefühle und Motive der Figuren ernst zu nehmen, sondern auch und vor allem den Willen, ihre unterstellten Begierden und Wünsche zu erfüllen, ihre Probleme zu lösen oder ihnen zu einem >besseren< Ende zu verhelfen als ursprünglich für sie vorgesehen war. ${ }^{20}$

Auch wenn das Konzept des >Fans man diese enthusiastische Einstellung zu literarischen Texten und die empathische Beziehung zu ihren Figuren als Vorläufer moderner Fankulturen auffassen. ${ }^{21}$ Fans sind Personen, die ihrem Gegenstand ein so uneingeschränktes Interesse entgegenbringen, dass sie bereit sind, alle damit verbundenen Informationen, Details, Nebenprodukte und Randerscheinungen unterschiedslos zur Kenntnis zu nehmen.22 Die Verfasser von Fanfiction rechnen mit diesem selektionslosen Interesse, wenn sie die Leserinnen und Leser mit Hintergrundgeschichten oder psychologischen Einblicken in die Charaktere versorgen, die in der ursprünglichen Geschichte nicht vorkamen. Sie können, müssen aber nicht in allen Fällen selbst auch Fans im engeren Sinn sein. Um 1800 wie heute wird Fanfiction gleichermaßen mit und ohne kommerzielle Absichten, von professionellen Autoren wie Amateuren geschrieben. ${ }^{23}$

In ihrem Verhältnis zum Ausgangstext und dessen Protagonisten unterscheidet sich Fanfiction auch von älteren Formen der Fortführung von fremder Hand, wie

17 Vgl. Matthew H. Birkhold, Characters before Copyright. The Rise and Regulation of Fan Fiction in Eighteenth-Century Germany, Oxford 2019, 12.

18 Vgl. ebd., $42 \mathrm{ff}$.

19 Erich Schön, »Geschichte des Lesens«, in: Bodo Franzmann u.a. (Hrsg.), Handbuch Lesen, München 1999, 1-85, hier: 31. Siehe auch Robert Darnton, »Readers respond to Rousseau«, in: Ders., The Great Cat Massacre and Other Episodes in French Cultural History, London 2001, 215-256.

20 Vgl. Henry Jenkins, Textual Poachers. Television Fans \& Participatory Culture, London 1992, 24.

$21 \mathrm{Zu}$ einer solchen Historisierung des Fanbegriffs vgl. Daniel Cavicchi, »Fandom Before >Fan<: Shaping the History of Enthusiastic Audiences«, Reception: Texts, Readers, Audiences, History 6 (2014), 52-72.

22 Das prominenteste Beispiel einer solchen frühen Fankultur sind vermutlich die Werther-Artikel (u.a. Fächer, Schlüsselanhänger, Parfums, Kleidungsstücke oder Geschirr), die begeisterte Leserinnen und Leser von Goethes Roman erwerben konnten. Vgl. den Katalog Die Leiden des jungen Werthers. Goethes Roman im Spiegel seiner Zeit. Eine Ausstellung des Goethe-Museums Düsseldorf, hrsg. Jörn Göres, Düsseldorf 1972.

23 Vgl. Birkhold (Anm. 17), 9. 
sie beispielsweise zu den Episodenromanen des 17. und frühen 18. Jahrhunderts verfasst wurden. Während etwa die Fortsetzungen galanter Romane in der Regel in einem konkreten öffentlichen Kommunikationszusammenhang situiert waren und aufgrund ihrer Referenz auf außertextuelle »Interaktions- und Kommunikationssituationen« oft eher ein loses Verhältnis zur Vorlage aufwiesen, ${ }^{24}$ ist Fanfiction selbst da, wo sie sich gegen die Intention des Autors oder der Autorin richtet programmatisch der Treue zu den Charakteren verpflichtet. Fanfiction, so die These, erkundet auf unterschiedlichen Ebenen die unrealisierten Möglichkeiten literarischer Werke. Sie stellt die Zwangsläufigkeit von Ereignisverläufen infrage und entwirft Alternativen zu dominanten Welterklärungsmodellen. Sie unterläuft zudem zeitgenössische Publikationsordnungen, indem sie nicht nur Veröffentlichungsformate jenseits des offiziellen Buchmarkts nutzt, sondern auch Genres und Erzählverfahren in unkonventioneller Weise kombiniert. Sie behandelt populäre Unterhaltungsliteratur wie kanonische Werke, die wiederholt und genau gelesen werden und einen Fundus gleichsam apokrypher Nebentexte hervorbringen; und sie macht kanonische Werke umgekehrt zum Gegenstand von Populärkultur und entzieht sie der Deutungshoheit ihrer Verfasser. ${ }^{25}$ Schließlich zeichnet sich Fanfiction dadurch aus, dass sie bei all dem häufig in der Interaktion mit anderen Texten steht: mit anderen fremden Aneignungen, von denen sie sich abgrenzt oder die sie sich zum Vorbild nimmt und bisweilen auch mit der ursprünglichen Vorlage, auf deren Fortführung sie sich auswirken kann. ${ }^{26}$

Damit sind zugleich die Potenziale der literaturwissenschaftlichen Erforschung von Fanfiction benannt, die im Bereich der deutschsprachigen Literatur noch weitgehend aussteht. ${ }^{27}$ Eine Ausnahme stellt Matthew Birkholds grundlegende Studie Characters before Copyright. The Rise and Regulation of Fan Fiction in EighteenthCentury Germany dar. ${ }^{28}$ Birkhold verwendet den Begriff Fanfiction bewusst anachronistisch, um dadurch einen neutralen Sammelbegriff für unterschiedliche Formen der literarischen Bearbeitung, Fortsetzung und Nachahmung um 1800 zu gewinnen. Während seine Studie allerdings in erster Linie einen Beitrag zu einer vergessenen Geschichte des geistigen Eigentums leisten möchte, ${ }^{29}$ steht im Folgenden stärker die Frage nach der Leistung von Fanfiction im Hinblick auf die Konstitution alternativer Wirklichkeiten im Vordergrund.

\footnotetext{
${ }^{24}$ Dirk Rose, Conduite und Text. Paradigmen eines galanten Literaturmodells im Werk von Christian Friedrich Hunold (Menantes), Berlin, Boston 2012, $155 \mathrm{ff}$.

25 Vgl. Jenkins (Anm. 20), 13.

26 Vgl. Kristina Busse, »Fan Fiction Tropes as Literary and Cultural Practices«, in: Sebastian Böck u.a. (Hrsg.), Lesen X.O. Rezeptionsprozesse in der digitalen Gegenwart, Göttingen 2017, 127-143.

27 Vgl. die Überblicksartikel von Petra Martina Baumann, »Halbe Literatur(en). Fanfiction als literaturwissenschaftliches und soziologisches Phänomen«, LiTheS. Zeitschrift für Literatur- und Theatersoziologie 2 (2009), 104-113; Judith Klinger, »Fan Fiction. Spielräume alternativer Wirklichkeiten«, in: Stephan Porombka, Susanne Scharnowski (Hrsg.), Phänomene der Derealisierung, Wien 1999, 93-116; Anne Neuschwander, »Wenn Begeisterung kreativ wird. Fanfiction - eine Domäne für Hobbyschreiber«, TextArt. Magazin für kreatives Schreiben 1 (2006), 34-39.

28 Vgl. Birkhold (Anm. 17).

29 Ebd., $13 \mathrm{f}$.
} 
Diese Frage wird am Beispiel von Vulpius' Roman Rinaldo Rinaldini untersucht, der schon kurz nach seiner ersten Veröffentlichung und noch vor dem Abschluss der Geschichte durch den Autor adaptiert, nachgeahmt und fortgesetzt wurde. ${ }^{30} \mathrm{Wie}$ sich zeigen wird, arbeitet die Fanfiction zum Rinaldini-Roman in unterschiedlicher Weise daran, jene Probleme, die im Original ungelöst bleiben oder deren Lösungen immer wieder aufgeschoben werden, zu beheben. Die fremden Aneignungen der Geschichte suchen nach Wegen, um das rastlose und getriebene Leben des Protagonisten zu befrieden. Die nicht enden wollende Kette abenteuerlicher, kontingenter Ereignisse wird unterbrochen, indem die Prinzipien von Zufall und Willkür jeweils zugunsten eines planvollen und zweckmäßigen Handlungsverlaufs aufgehoben werden. Die Fanfiction zum Roman zeigt, dass sich Rinaldo Rinaldini keineswegs bloß als episodischer Abenteuerroman lesen lässt, sondern ebenso als romantische Liebesgeschichte oder als Erziehungsroman interpretiert und fortgeschrieben werden kann. Die Gattungsfrage wird zur Lektürestrategie. ${ }^{31}$

An Vulpius' Räubergeschichte lässt sich beobachten, dass der Produktion von Fanfiction häufig idiosynkratische Lesarten zugrunde liegen, die einzelne Merkmale, Stellen oder Handlungsstränge des Quellentextes als so zentral deuten, dass andere Aspekte darüber vollständig ausgeblendet werden. ${ }^{32}$ Und der Roman zeigt zugleich, dass es Erzählstrukturen gibt, die solche eigenwilligen Lektüren in besonderer Weise begünstigen. Die hybride Anlage und die offene Struktur des Rinaldo Rinaldini also jene Merkmale, die von der Kritik verurteilt wurden - sind in Kombination mit dem Entwurf eines melancholischen Hauptcharakters aus dieser Perspektive geradezu der Grund für die intensive und produktive Art seiner Rezeption: Einerseits ist das Erzählgefüge bei Vulpius so locker gestrickt, dass im Hinblick auf einen möglichen Abschluss des Romans keine Zwangsläufigkeit besteht. ${ }^{33}$ Die disparaten Episoden, die durch die Erzählinstanz ohne kausal- oder finalgenetische Verknüpfungen aneinandergereiht sind, werden von der Hauptfigur immer wieder als Ereignisse eines unberechenbaren Zufalls gedeutet. Von den Verfasserinnen und Verfassern der Fanfiction können sie daher in ganz unterschiedlicher Weise auf ein Ende hingeführt werden. Andererseits macht der Roman eine ganze Reihe von Angeboten, worin ein mögliches Telos der Geschichte bestehen könnte. In der Fanfiction werden einige dieser losen Enden aufgegriffen und weitergesponnen. Rinaldini kann hier die Rehabilitation erhalten, die ihm sein Verfasser verwehrt hatte, zu einem glücklichen

\footnotetext{
${ }^{30}$ Auf die reichhaltige Fanfiction zu Vulpius' Roman macht bereits Birkhold (Anm. 17), 154f., aufmerksam.

${ }^{31}$ Henry Jenkins spricht in diesem Zusammenhang von den Verfasserinnen und Verfassern von Fanfiction als Wilderern (Poachers), die bloß diejenigen Elemente der Quellentexte zur Kenntnis nehmen, die sie gebrauchen können. Vgl. Henry Jenkins, Textual Poachers. Television Fans \& Participatory Culture, London 1992, 24.

32 Peter J. Rabinowitz, »The Turn of the Glass Key: Popular Fiction as Reading Strategy«, Critical Inquiry 11/3 (1985), 418-431.

33 Dass dies für episodisches Erzählen charakteristisch ist und die Enden von Reihenromanen oder Serien daher oft »bombastisch inszenierte Schlüsse von geradezu operettenhafter Dramatik « aufweisen, weil hier »all jene narrativen Verknüpfungen geleistet werden «, die zuvor nur ansatzweise vorhanden sind, betont Florian Kragl, »Episodisches Erzählen - Erzählen in Episoden. Medientheoretische Überlegungen zur Systematik, Typologie und Historisierung «, Diegesis 6/2 (2017), 176-197, hier: 184.
} 
Ehemann werden und doch noch den Zweck hinter all seinen Erlebnissen entdecken. Mit derartigen Aneignungen hat die Fanfiction zum Roman nicht zuletzt auch Vulpius' eigene Fortsetzung der Geschichte beeinflusst - ein Umstand, der von der Forschung bisher unbemerkt geblieben ist.

\section{II.}

Das Vorwort zum ersten Band des Rinaldo Rinaldini weist den Roman als Teil einer mündlichen Erzählkultur aus:

Ganz Italien spricht von ihm [...]. Die geschwätzigen Stadtbewohner Calabriens versammeln sich Abends vor ihren Häusern, und jeder in der Versammlung weiß ein Geschichtchen von dem valeroso Capitano Rinaldini zu erzählen. [...] Die Hirten in Siziliens Thälern unterhalten sich wechselseits mit Rinaldini's Abenteuern, und der einsylbige Landmann, der des Tages Last und Hitze trug, wird belebt, wenn er des Abends im Zirkel seiner Bekannten von Rinaldini sprechen kann. ${ }^{34}$

Vor diesem Hintergrund präsentiert sich der Verfasser als bloßer Herausgeber, der die Abenteuer »geordnet « und in eine chronologische Reihenfolge gebracht habe, sodass sich daraus die »Geschichte « Rinaldinis im Singular ergibt. ${ }^{35}$ Auf dieser Verknüpfung zwischen Folklore und Literatur, ${ }^{36}$ zwischen einer episodischen und einer linearen Erzählstruktur beruht die Grundkonzeption des Romans. Schon auf den ersten Seiten zeigt sich, dass die Dynamik des Geschehens wesentlich von dem Konflikt getragen wird, der sich aus der zirkulären Logik der Ereignisse einerseits und der Sehnsucht des Protagonisten nach Veränderung und Fortschritt andererseits ergibt.

Die Geschichte setzt mit einer Begegnung ein, die scheinbar alles verändert: Der Räuberhauptmann verliebt sich in die schöne Aurelia, die Tochter des Prinzen della Rocca, und beginnt dadurch sein gesetzloses Dasein zu hinterfragen. Die »Unschuld « des Mädchens weckt in ihm den Wunsch, seiner bisherigen »Lebensart « gänzlich zu »entsagen «. ${ }^{37}$ Als Aurelia allerdings von ihrem Vater fortgebracht und mit einem anderen Mann verheiratet wird, wendet er sich, obwohl er immer wieder seufzend an die unerfüllte Liebschaft zurückdenkt, kurzerhand der nächsten Dame zu: In der Reihe der Liebhaberinnen folgen Rosalie, Olimpia, Dianora, Serena und viele andere. Die Frauen werden für Rinaldini regelmäßig zum Anker einer Ausstiegsphantasie, die sich immer wieder aufs Neue als unrealisierbar erweist. Der verwitweten Gräfin Dianora de Martagno kommt dabei in gewisser Weise eine Son-

\footnotetext{
34 [Christian August Vulpius], Rinaldo Rinaldini, der Räuberhauptmann. Eine romantische Geschichte unseres Jahrhunderts. Erster Theil, neue, durchgesehene und verbesserte Auflage, Leipzig 1801, IIIf.

35 Ebd., VI.

36 Zum Verhältnis von Literatur und Folklore siehe Aleida Assmann, »Schriftliche Folklore. Zur Entstehung und Funktion eines Überlieferungstyps«, in: Aleida Assmann, Jan Assmann, Christof Hardmeier (Hrsg.), Schrift und Gedächtnis. Archäologie der literarischen Kommunikation I, München 1983, 175-193.

37 Ebd., 39.
} 
derstellung zu. Rinaldini trifft sie im zweiten Teil des Romans in Messina, wo er unter falschem Namen, als Ritter de la Cintra, in die vornehme Gesellschaft eingeführt wird. Zunächst schwankt sein Interesse zwischen Laura, einem »Fräulein von hoher Schönheit«, und Dianora, die als »Dame von Geist und dem feinsten einnehmenden Wesen« vorgestellt wird. ${ }^{38}$ Als sich Laura allerdings in geselliger Runde abfällig über den Räuberhauptmann Rinaldini äußert - nicht ahnend, dass er ihr in diesem Moment gegenübersitzt -, erhält Dianora den »ganzen Platz in Rinaldo's Herzen «. ${ }^{39}$ Ein Spaziergang führt ihn einige Tage später zufällig zum Landsitz der Gräfin und die beiden beginnen eine Liebesbeziehung.

Erstmals gestört wird das Liebesidyll, als Rinaldini von einem seiner Feinde, einem korsischen Kapitän, aufgespürt und konfrontiert wird. Es kommt zum Kampf, der Kapitän wird von einem Anhänger Rinaldinis schwer verwundet und Rinaldini flieht auf ein weiteres Schloss der Gräfin, das abseits im Gebirge liegt. Dort wird das Liebesverhältnis fortgesetzt, bis die schwangere Dianora schließlich Rinaldinis wahren Namen erfährt und er sie verlässt. Rinaldini irrt daraufhin weiter durch die Welt, begegnet erneut Laura, Olimpia und Rosalie und beginnt schließlich ein Verhältnis mit der Kammerjungfer Serena. Mehrfach gerät er in Gefangenschaften, aus denen er von Freunden, unter anderem von dem geheimnisvollen »Alten von Fronteja «, befreit wird. Der »Alte« versucht vergeblich, Rinaldini für den korsischen Freiheitskampf zu begeistern und aus dem Räuberhauptmann einen Kriegshelden zu machen. Rinaldini aber will sich zur Ruhe setzen und flüchtet sich auf die Insel Pantelleria, wo er hofft, »unter guten, unverdorbenen, reinen Naturmenschen eine stille, friedliche Stätte zu finden $\ll^{40}$ Doch diese utopische Zwischenepisode scheitert schon daran, dass Rinaldinis sämtliche Weggefährten, von dem Alten über Olimpia und Serena bis zu Dianora, ebenfalls auf der Insel sind oder im Laufe seines Aufenthalts dort eintreffen. Gerade in dem Moment, als Dianora Rinaldini verzeiht und bereit ist, mit ihm auf den Kanarischen Inseln ein neues Leben zu beginnen, wird er von seinem Erzfeind, dem Kapitän, verraten. Bevor er allerdings festgenommen werden kann, wird er von dem geheimnisvollen Alten aus Gnade niedergestochen: »Rinaldo! - sagte er: - ich habe dir meine Freundschaft bis in den Tod versprochen. Ich halte Wort. Du bist nicht zu retten. Fahre wohl! $\ll^{41}$

Mit diesem tragischen Tod schien Rinaldinis Geschichte 1799 nach drei Teilen abgeschlossen zu sein. Dem Publikum missfiel dieses Ende aber offenbar - zumindest berichtete Vulpius von den Zuschriften begeisterter Leserinnen, die ihn bedrängten, den Räuberhauptmann noch einmal aufleben zu lassen und ihm ein glücklicheres Schicksal zu ermöglichen. ${ }^{42}$ Und auch der Verleger votierte angesichts des überraschenden Erfolgs des Romans und der Gefahr illegitimer Aneignungen für eine

\footnotetext{
38 [Christian August Vulpius], Rinaldo Rinaldini, der Räuberhauptmann. Eine romantische Geschichte unseres Jahrhunderts. Zweyter Theil, neue, durchgesehene und verbesserte Auflage, Leipzig 1801, 38.

39 Ebd., 46.

40 [Christian August Vulpius], Rinaldo Rinaldini, der Räuber-Hauptmann. Eine romantische Geschichte unseres Jahrhunderts, Dritter Theil, Vierte, verbesserte Auflage, Leipzig 1802, 156.

41 Ebd., 227.

42 Vgl. [Christian August Vulpius], Ferrandino. Fortsetzung der Geschichte des Räuberhauptmanns Rinaldini von dem Verfasser desselben, Leipzig 1801, V.
} 
rasche Fortsetzung. ${ }^{43}$ Doch bevor Vulpius diesem Wunsch nachkommen konnte, ${ }^{44}$ erschien noch im Jahr 1799 im Leipziger Joachimschen Literarischen Magazin ein Roman, der nicht nur Vulpius' Version abwandelte und fortsetzte, sondern vor allem eine der Nebenfiguren ins Zentrum rückte: Das »romantische Gemählde« Dianora, Gräfin Martagno, Rinaldo Rinaldinis Geliebte schildert die Ereignisse aus der Perspektive Dianoras und kommt dabei zu anderen Schlüssen. ${ }^{45}$

Der Roman wurde anonym veröffentlicht und als »Anhang zu Rinaldo Rinaldini« beworben - die Erstleserinnen und -leser konnten also durchaus der Täuschung erliegen, Vulpius selbst habe den Text verfasst. Für den eigentlichen Verfasser, den wohlhabenden Leipziger Notar und Anwalt Johann Jakob Brückner, war diese Form des literarischen Trittbrettfahrens keine Ausnahme: Sein Werk besteht zu großen Teilen aus Fortsetzungen, sogenannten Gegen- oder Seitenstücken zu den Texten prominenter Autoren wie Schiller, Bürger, Stolberg oder Spieß. ${ }^{46}$ Auch dass Brückner sich für eine Zusammenarbeit mit dem Verleger Gottfried Andreas Joachim entschied, ist keine Überraschung: Joachim, der neben seinem Verlags- und Sortimentsbuchhandel auch ein Lesekabinett in Leipzig unterhielt, war berüchtigt dafür, die Publikationen anderer Verleger nachzudrucken. ${ }^{47}$ Um seine Aktivitäten zu verschleiern, verstieß er gegen die Impressumspflicht und benutzte insgesamt mindestens vier unterschiedliche Firmenbezeichnungen. ${ }^{48}$ Fanfiction, die mit den Namen populärer Protagonisten auf den Titelblättern warb und deren Geschichten fortsetzte oder umschrieb, war anders als der Nachdruck von Büchern - zwar nicht offiziell verboten, stellte aber doch eine Verletzung informeller Rechtsgepflogenheiten dar und war öffentlicher Kritik ausgesetzt. ${ }^{49}$ Bei Brückners Roman kommt hinzu, dass er eine ganze Reihe von Szenen, die auch bei Vulpius vorkommen, aufgreift und teilweise wörtliche Zitate aus den Dialogen übernimmt, also durchaus als Teilplagiat gewertet werden konnte.

\footnotetext{
${ }^{43}$ In seiner Anzeige der zweiten Auflage kündigte der Verleger an: »Der Verfasser wird eine Fortsetzung liefern. Wie dies möglich ist, mögen die ergrübeln, welche Rinaldo gelesen haben. Glückauf, Rinaldo!!« [Heinrich Gräff], »Anzeige von neuen Auflagen einiger interessanten Schriften«, Intelligenzblatt der Allgemeinen Literatur-Zeitung, Nr. 128, Mittwochs den 9ten October 1799, 1039-1040, hier: 1040.

${ }^{44}$ Die Fortsetzung erschien 1800 unter dem Titel Ferrandino. Fortsetzung der Geschichte des Räuberhauptmanns Rinaldini.

45 [Johann Jakob Brückner], Dianora, Gräfin Martagno, Rinaldo Rinaldinis Geliebte. Ein romantisches Gemälde in zwey Theilen oder acht Büchern, Anhang zu Rinaldo Rinaldini, Leipzig [1800].

${ }^{46} \mathrm{Vgl}$. den Eintrag »Johann Jacob Brückner« im Grundriss zur Geschichte der deutschen Dichtung aus den Quellen von Karl Goedeke, zweite, ganz neu bearbeitete Auflage, nach dem Tode des Verfassers in Verbindung mit Fachgelehrten fortgeführt von Edmund Goetze, Bd. V, Sechstes Buch: Vom siebenjährigen bis zum Weltkriege, Berlin 2011, 533; zu Brückners nur spärlich erforschter Biografie siehe auch Wolfgang Griep, »Johann Jakob Brückner«, in: Killy Literaturlexikon, Bd. 2, 2. Aufl., Berlin 2008, 225.

47 Vgl. Michael Wögerbauer, »Geheime Wege nach Leipzig? Die Entstehung der Berufsschriftstellerei in den Böhmischen Ländern und die österreichische Zensur«, in: Geheimliteratur und Geheimbuchhandel in Europa im 18. Jahrhundert, hrsg. Christine Haug u.a., Wiesbaden 2011, 185-201, hier: $196 \mathrm{ff}$.

48 Ebd., 196. Vgl. auch Helge Buttkereit, Zensur und Öffentlichkeit in Leipzig 1806 bis 1813, Berlin 2009, 54.

49 Zur rechtlichen Diskussion über Fanfiction um 1800 siehe Birkhold (Anm. 17), 115 ff. Zu den informellen Regeln, die die Produktion von Fanfiction bestimmten, zählt Birkhold u.a. das Einverständnis bzw. das Wissen des Verfassers und die korrekte Titulierung - d.h. es musste deutlich gemacht werden, dass es sich bei der Fanfiction nicht um eine Fortsetzung des Erstautors handelt.
} 
Verleger wie Autor partizipierten somit am Erfolg des Rinaldo Rinaldini, indem sie für die Publikation ihrer Fanfiction eine rechtliche Grauzone nutzten. Allerdings gehen Brückners Aneignungen fremder literarischer Figuren und Geschichten nicht in diesem ökonomischen Profitinteresse auf. ${ }^{50}$ Dianora erzählt Vulpius' Räubergeschichte nicht nur aus einer anderen Perspektive, sondern weicht vor allem an entscheidenden Stellen von der Vorlage ab. Der Roman zeugt gleichermaßen von einer genauen Lektüre des Rinaldo Rinaldini wie auch von dem Bestreben, alternative Antworten auf die bei Vulpius ungelösten Probleme zu geben.

Dass Dianora sich nicht bloß als Gegenstück, sondern auch in mancher Hinsicht als Gegenentwurf zum Rinaldo Rinaldini versteht, zeigt bereits ein Vergleich der Romananfänge, die jeweils mit einer Beschreibung der Wetter- und Stimmungslage einsetzen. Bei Vulpius stehen alle Zeichen auf Unruhe und Chaos: »Stürmisch brausete der Wind, tobend wie empörte Meereswogen, über den Nacken der hohen Alpenninen [...].« Rinaldini nimmt den Sturm nicht nur »hier und dort, um uns« und »neben uns « wahr, sondern auch »in mir, und überall «. ${ }^{11}$ Brückners metereologisches Stimmungsbarometer zeigt demgegenüber eine optimistischere Gemütslage an: »Es war ein schöner Tag. Mit sehnlichem Verlangen lauschte alles dem vierten Glockenschlage entgegen, der die Schönen der Stadt und die anwesenden Ritter und Noblen zu einer glänzenden Fete versammeln sollte. ${ }^{52}$ Der Roman setzt unmittelbar mit der Begegnung zwischen Dianora und Rinaldini ein. Anders als bei Vulpius ist der Beginn dieses Liebesverhältnisses kein zufälliges Ereignis, das sich in Rinaldinis Leben vielfach wiederholt, sondern eine schicksalhafte Begegnung.

Wie im Original ist der Ritter de la Cintra alias Rinaldini auch in Brückners »Anhang « zunächst hin- und hergerissen zwischen Dianora und Laura, und auch hier sticht Dianora ihre Nebenbuhlerin erst aus, als diese den Räuber Rinaldini in der Abendgesellschaft als »gottlosen « Verbrecher verurteilt. ${ }^{53}$ Dann weichen die beiden Fassungen jedoch voneinander ab. Wo Vulpius seinen Helden als Getriebenen darstellt, der beinahe ohne sein eigenes Zutun von einem Abenteuer ins nächste gerät, macht Brückner ihn wieder zum Herrn seiner eigenen Geschichte. Brückner streicht, mit anderen Worten, den unkalkulierbaren Faktor Zufall aus dem Roman heraus. Bei Vulpius geht Rinaldini nach dem Ball am nächsten Morgen außerhalb der Stadt in der »Gegend der Gärten und Landhäuser« spazieren. Dort träumt er davon, in ein »fernes Land « auszuwandern und als Hirte ein einfaches Leben $\mathrm{zu}$ leben. ${ }^{54}$ Wenige Tage später ist er wieder in derselben Gegend unterwegs und betritt zufällig den Garten, der zum Landhaus der Gräfin Dianora gehört. Während Rinaldini »unentschlossen« ist, ob er gehen oder bleiben soll, spricht die Gräfin ihn an:

\footnotetext{
50 Dagegen spricht schon, dass Brückner finanziell gut situiert und nicht auf schriftstellerische Nebeneinkünfte angewiesen war, vgl. Gustav Siechelschmidt, Liebe, Mord und Abenteuer. Eine Geschichte der deutschen Unterhaltungsliteratur, Berlin 1969, 94.

51 [Vulpius], Rinaldo Rinaldini, Erster Theil (Anm. 34), 7.

52 [Brückner] (Anm. 45), 5.

53 Ebd., 10.

54 [Vulpius], Rinaldo Rinaldini, Zweyter Theil (Anm. 38), 48.
} 
»Ritter! Darf ich meinen Augen trauen? Seyd ihr es selbst, oder ist es Euer Geist?« [...] »Um aller Heiligen willen!« fuhr die Gräfinn fort, »wie habet Ihr meine Villa gefunden?«

Er. Wie man oft mehr in der Welt, als eine Villa findet, durch Zufall.

Sie. Ihr hättet doch ein wenig galanter seyn, und mich nicht zur Schuldnerinn des bloßen Zufalles machen sollen.

Er. Die Schuld wird nicht groß seyn.

Sie. Wenigstens eben so groß, als euer Zufall.

Er. So sind wir ja quitt. ${ }^{55}$

Die Zufälligkeit der Begegnung missfällt der Gräfin nicht zuletzt deshalb, weil Rinaldini, wie sie bemerkt, mit ein paar weiteren Schritten ebenso zufällig beim »Fräulein Laura« hätte landen können. Diese Logik, die sehr präzise beschreibt, wie die Ereignisse und die romantischen Begegnungen bei Vulpius aneinander angeschlossen werden, wird bei Brückner durch eine planvolle Zusammenkunft der Liebenden ersetzt.

In Dianora sucht Rinaldini die Gräfin gleich am nächsten Morgen nach dem Fest in ihrer Stadtvilla auf und erfährt von einem Bedienten, dass sie ihn bereits auf ihrem Landsitz erwarte. Rinaldini verirrt sich auf dem Weg, landet bei der falschen Villa und kommt erst abends am Haus der Gräfin an, die bereits den ganzen Tag vergeblich auf ihn gewartet hat. Das anschließende Gespräch wirkt wie ein Kommentar zu dem Dialog, der bei Vulpius zwischen Rinaldo und Dianora stattfindet:

Um Himmels willen, Ritter! seyd ihr es selbst, oder ist's euer Geist? [...] Warum so spät Ritter?«

Er. Ein Werk des Zufalls, Gräfin!

Sie. War ich nicht schon diesen Morgen hier?

Er. Verzeiht!

Sie. Wollt ihr mich Lügen strafen?

Er. Das nicht; aber - euer Bedienter sezte wahrscheinlich voraus, daß ich den Ort Eures Aufenthalts wüßte.

Sie. Und ihr giengt fehl, nicht wahr?

Er. So ists.

Sie. Ihr seyd zu bescheiden, mir die Schuld beizumessen. Ich danke euch, Ritter! und bleibe eure Schuldnerin. ${ }^{56}$

Während die Zusammenführung des Liebespaars bei Vulpius von der Erzählinstanz als reine Zufallsbegegnung dargestellt und von den Figuren auch so gedeutet wird, sorgt er bei Brückner lediglich für eine Verzögerung des ohnehin geplanten Treffens. Wird der originale Rinaldo von einem ungerichteten Begehren angetrieben, das je nach Zufallsbegegnung von einer anderen Frau gestillt wird, kennt sein Nachfolger immer schon das Ziel seiner Sehnsucht, an dem er lediglich durch die Schuld des Zufalls - etwa durch eine ungenaue Adressangabe - etwas verspätet eintrifft.

55 Ebd., $50 \mathrm{f}$.

56 [Brückner] (Anm. 45), 16f. 
Bei Brückner werden die Figuren nicht mehr als Spielbälle sinnloser Ereignisketten durch die Welt getrieben, sondern sie betreiben gezielte Kontingenzreduktion, indem sie Pläne schmieden, Entscheidungen treffen und überdies an schicksalhafte Fügungen glauben. Brückner schildert zwar eine ähnliche Ereignisfolge wie Vulpius, lässt sie die Figuren aber anders interpretieren und zeigt damit, dass die Umwandlung von Zufall in Schicksal oft eine Frage der Haltung ist. Das unendliche Spektrum der Möglichkeiten wird begrenzt, indem Alternativen schlicht ausgeschlossen werden. Ist Dianora in Vulpius' Roman bis zuletzt eine Geliebte unter vielen, die bei jeder zufällig herbeigeführten Trennung durch eine andere Frau ersetzt werden kann, so setzt Brückners Rinaldo auf Exklusivität: »[E]r fand in ihr eine Dame von Geist, der er vor allen andern den Vorzug verstattete und fühlte eine mehr als gewöhnliche Neigung für sie, ein süßes Gemengsel von Liebe und Hochachtung. « ${ }^{57}$ Bei Brückner ist die Liebe nicht mehr wie bei Vulpius ein beliebig oft wiederholbares Produkt des »Zufalls« der Begegnung. ${ }^{58}$ Sie wird vielmehr zur »Kontingenzformel«, die eine begründete Selektion ermöglicht, »obwohl - oder: gerade weil - auch anderes möglich ist «: Indem Dianora und Rinaldini sich begegnen, fallen »Zufall und Notwendigkeit zusammen «. ${ }^{59}$ Der »Augenblick « der »Verkettung « ihrer Schicksale wird zum »seligeste[n] ihres Lebens «. ${ }^{60}$ Das »günstige Schicksal« und der »glückliche Zufall« treten im Roman stets gekoppelt auf. ${ }^{61}$

Dass Brückner den Rinaldo Rinaldini auf das Liebesverhältnis zwischen Dianora und Rinaldo reduziert, hat aus dieser Sicht eine größere Tragweite als bloß die Umwandlung eines Abenteuerromans in eine romantische Liebesgeschichte. Der Perspektiv- und Gattungswechsel impliziert zugleich eine veränderte Haltung zum Problem des Zufalls: Während der Zufall bei Vulpius das Prinzip ist, das den Verlauf der Ereignisse im Positiven wie Negativen bestimmt, ist er bei Brückner lediglich ein Störfaktor, der zwar für Abweichungen innerhalb eines sinnhaften Handlungszusammenhangs sorgen, aber die providenzielle Logik der Geschichte nicht nachhaltig irritieren kann. Brückner entwirft in seiner Fassung des Romans eine alternative Welt, nur um zu zeigen, dass es zum Glauben an den schicksalhaften Lauf der Ereignisse keine Alternativen gibt.

Plausibilisiert wird diese providenzielle Logik auch durch eine verfeinerte Figurenpsychologie. Brückners Rinaldini ist entscheidungsfreudiger und zugleich reflektierter als seine literarische Vorlage. Diese Differenz wird durch Fußnotensetzungen, die auf einzelne Stellen in Vulpius' Roman hinweisen, gezielt markiert. Kurz bevor Dianora Rinaldos wahre Identität herausfindet, findet im Original ein Gespräch zwischen den beiden statt, in dem der Räuberhauptmann seiner Geliebten lapidar mitteilt, dass er sie verlassen werde:

\footnotetext{
57 Ebd., 19.

58 Zur Bedeutung des Startmechanismus Zufall für die romantische Liebe, der als »Notwendigkeit«, $»$ Schicksal« oder »Freiheit der Wahl« »paradoxiert« wird, siehe Niklas Luhmann, Liebe als Passion. Zur Codierung von Intimität, Frankfurt a. M. 1982, $180 \mathrm{f}$.

59 Niklas Luhmann, »Einführende Bemerkungen zu einer Theorie symbolisch generalisierter Kommunikationsmedien«, in: Ders., Soziologische Aufklärung 2, Opladen 1975, 230.

60 [Brückner] (Anm. 45), 21.

61 Ebd., 256.
} 
Er. [...] Ich gedenke Sicilien zu verlassen.

Sie. Allein?

Er. Wer sollte mit mir gehen, als mein Ludovico?

Sie. Dieser nur allein?

Nur er. Er verläßt mich nicht. ${ }^{62}$

Erst nach mehrfachem Nachfragen kommt Dianora der Verdacht, dass »la Cintra « nicht der »wahre Nahme« ihres Geliebten ist. Sie insistiert so lange, bis Rinaldo schließlich seine Identität preisgibt.

Bei Brückner geht diesem Gespräch ein seitenlanger innerer Monolog Rinaldos voraus, in dem er bekennt, mit Dianora zwar sein »höchstes Glück« gefunden zu haben, sich »mit jedem Augenblicke« aber »unwürdiger« zu fühlen, »den Kelch zu leeren, den die edelste der Frauen ihm darreichte. ${ }^{63}$ Der Entschluss, Dianora zu verlassen, steht dementsprechend auch nicht am Anfang des Gesprächs, sondern in dessen Mitte. Er ist erst die Konsequenz eines längeren Dialogs, in dem Rinaldos innerer Konflikt dargelegt wird: Wiederholt betont er, dass seine Gefühle für die Geliebte »unvertilgbar« seien, er jedoch fürchte, sie durch seine Liebe zu »tödten«. Brückner zeigt diese Abweichung von der Vorlage durch die Anmerkung »vergl. Mit Rinaldo c. 5s B. S. 316.« eigens an und macht so darauf aufmerksam, dass seine Fassung die komplexen psychologischen Beweggründe für das Verhalten der Hauptfigur nachliefert, die bei Vulpius fehlen. ${ }^{64}$ Was im Original wie ein spontaner Entschluss des Protagonisten anmutet, der auf die Gefühle der Geliebten kaum Rücksicht nimmt, wird so zum Resultat eines langwierigen inneren Konflikts. Bei Brückner ist Dianora mehr als nur eine Zwischenstation auf einer abenteuerlichen Reise. Sie ist das Ziel seiner Odyssee, das ihm durch die Unmöglichkeit einer ehelichen Verbindung jedoch vorerst noch verwehrt ist.

Brückner verleiht dem Räuberhauptmann Rinaldini damit jene psychologische Tiefe, die Blanckenburg gefordert hatte, um dem Publikum die Motive für die Handlungen der Figuren nachvollziehbar zu machen, und stattet den Roman zugleich mit einem Telos aus. Angesteuert wird dieses Telos durch eine narrative Strategie der Restlosigkeit, die auf allen Ebenen umgesetzt wird: Während das Schicksal den Zufall als Deutungsmuster der Figuren innerhalb der Erzählung ablöst und Rinaldinis Welt wieder sinnhaft erscheint, während die kleinteilige psychologische Introspektion keine Zweifel mehr an den Gefühlen, Motiven und dem Begehren der Protagonisten lässt, werden auf der Handlungsebene alle Geheimnisse und offenen Fragen uneingeschränkt aufgeklärt. Das betrifft etwa Ereignisse wie das Rätsel um eine in Dianoras Schloss eingekerkerte Frau namens Violante, die sich nach ihrer Befreiung mit der Gräfin anfreundet. Während die Umstände der Gefangenschaft bei Vulpius nur teilweise aufgeklärt werden, gibt es bei Brückner eine ganze Reihe zusätzlicher

\footnotetext{
62 [Vulpius], Rinaldo Rinaldini, Zweyter Theil (Anm. 38), 77.

63 [Brückner] (Anm. 45), $93 \mathrm{f}$.

64 Ebd., 94; diese Verweise auf Vulpius bemerkt auch Birkhold (Anm. 17), 191.
} 
Szenen, die die Hintergründe von Violantes Schicksal erklären. ${ }^{65}$ Jenseits der Entschlüsselung solcher Mysterien identifiziert Brückner aber vor allem eine zentrale Leerstelle in Vulpius' Roman, die er nutzt, um Rinaldos rastlose Irrfahrt zu einem Ende zu bringen: die Herkunft des Räuberhauptmanns. Mit dieser Deutung beeinflusste Brückner nicht nur Vulpius' Fortsetzung des Romans, sondern regte indirekt auch die Produktion weiterer Fanfiction an.

\section{III.}

Bei Vulpius erfährt man über Rinaldos Kindheit lediglich, dass er als jüngstes von sechs Kindern die Ziegen seiner Eltern hütete und dabei die Gesellschaft eines Eremiten namens Onorio suchte. Onorio bringt ihm Lesen und Schreiben bei, verschwindet aber »unvermutet «, als Rinaldo siebzehn Jahre alt ist. Rinaldo strebt schon früh nach Großem, liest Abenteuerromane und wird Soldat, um dort seine »Ideale« zu realisieren. Doch in seinem Regiment gerät er bald in »Händel«, ersticht seinen »Chef « und muss fliehen. Auf der Flucht begegnet er einer Räuberbande, die ihn bald zu ihrem Anführer macht. ${ }^{66}$

Rinaldos Weg in die Gesetzlosigkeit ist also einer Mischung aus mangelnder Affektbeherrschung, Schwärmertum und ungerichtetem Tatendrang geschuldet. ${ }^{67}$ Bei Vulpius wie bei Brückner wird immer wieder die Möglichkeit ins Spiel gebracht, dass Rinaldo unter anderen Bedingungen anstelle eines Räuberhauptmanns ein großer Kriegsheld und Heerführer hätte werden können. ${ }^{68}$ Brückners Roman gibt auf die Frage, welche Bedingungen das sein könnten, eine klare Antwort: Eine »endliche und glückliche Lösung « kann es für Rinaldos »Lebenskatastrophe« nur geben, indem er einen Vater findet. ${ }^{69}$ Während die glückliche Sozialisierung im zeitgenössischen Bildungsroman häufig gerade am Konflikt mit einem vorhandenen, aber problematischen Elternhaus scheitert und der individuelle Bildungsweg sich über die Emanzipation von der väterlichen Autorität realisiert, ${ }^{70}$ beschreitet Brückners Rinaldo den umgekehrten Weg. Die fehlgeleitete Biografie findet ihr Ziel in der Heimkehr.

Nachdem Rinaldo wie im Original auf der Insel Pantelleria niedergestochen wird, kann er sich in Brückners Fassung erholen und entkommen. Erneut wird er von Dianora und dem inzwischen geborenen Sohn getrennt. Schließlich landet die ganze Familie auf den Liparischen Inseln, wo es zu einer erneuten Konfrontation mit dem

\footnotetext{
65 Während etwa bei Vulpius im Dunkeln bleibt, wer Violante über Jahre hinweg in ihrem unterirdischen Gefängnis mit Essen versorgte, kommt es bei Brückner zu einer Konfrontation und einem Gespräch mit dem Kerkermeister, der sich als Gefolgsmann von Dianoras erstem, inzwischen verstorbenen Ehemann herausstellt, vgl. [Brückner] (Anm. 45), $81 \mathrm{ff}$.

66 [Vulpius], Rinaldo Rinaldini, Dritter Theil (Anm. 40), $193 \mathrm{f}$.

67 Dass Rinaldo zum Räuber vor allem »aufgrund mangelnder Affektkontrolle« wird, betont Simanowski (Anm. 10), 344.

68 Vgl. z.B. [Vulpius], Rinaldo Rinaldini, Zweyter Theil (Anm. 38), 46; [Brückner] (Anm. 45), 10.

69 [Brückner] (Anm. 45), 282.

$70 \mathrm{Vgl}$. Rolf Selbmann, Der deutsche Bildungsroman, 2. überarbeitete und erweiterte Auflage, Stuttgart 1994, $1 \mathrm{ff}$.
} 
korsischen Kapitän kommt, der diesmal von Dianora getötet wird. Rinaldo wird dem Liparischen Statthalter vorgeführt, dem er seine Lebensgeschichte erzählt. Der Statthalter, der Rinaldo offenbar sofort erkennt (»Er ist es!«), bringt ihn zum Pascha von Zypern. Dieser entpuppt sich als Rinaldos Vater, der seinen Sohn seit Jahren vergeblich gesucht hat: »Dies ist Dein Vater, Rinaldo! - Dieser da, Bassa, Euer Sohn. $\ll^{71}$ Mit dieser »glücklichen Wendung « von Rinaldos »Schicksal« ist nicht nur die Schande des Namens aus dem Weg geräumt, die eine eheliche Verbindung mit der Gräfin Martagno verhindert hatte, sondern vor allem realisiert sich Rinaldos eigentliche Bestimmung, zum militärischen Führer zu werden und damit an der Spitze eines »würklichen Kriegsheers « zu stehen. ${ }^{72}$ An der Seite des Pascha, der gleichermaßen die familiäre wie die staatliche Autorität repräsentiert, wird das Leben des einstigen Räuberhauptmanns wieder in seine vorbestimmten Bahnen geleitet. Brückners Antwort auf die Frage, wie das Abgleiten in eine »entehrende Lebensart « verhindert und eine Rückkehr in ein geregeltes Dasein erreicht werden kann, ${ }^{73}$ besteht also in der »Orientierung auf eine ständisch-geordnete Lebensführung «. ${ }^{74}$ Nicht der individuelle Bildungsweg führt Rinaldo ans Ziel, sondern die Integration in eine qua Geburt vorgezeichnete Laufbahn. Um doch noch eine »Entwicklung « durchzumachen und die perpetuierte Stagnation seines Lebenslaufs unterbrechen zu können, muss Rinaldo das »unerzählte Geheimnis « seines »Ursprungs « erkunden. ${ }^{75}$ So wie Rinaldinis vorherige Liebesabenteuer in dem Moment, als er Dianora begegnet, nur noch die Funktion haben, die Differenz zwischen falscher Erotik und wahrer Liebe anzuzeigen, erscheinen seine Räubertaten rückblickend als bloße Abweichungen vom einer insgeheim immer schon für ihn vorgesehenen militärischen Laufbahn.

Es ist bezeichnend, dass Vulpius, der die Aneignungen und Fortsetzungen seines Romans sehr genau beobachtet hat, ${ }^{76}$ in seiner eigenen, ein Jahr später erschienenen Fortsetzung Ferrandino Brückners >Lösungsvorschlag< in modifizierter Form aufgreift. Auch bei Vulpius finden sich Dianora samt Kind und Rinaldini auf den Liparischen Inseln wieder, und auch hier tötet Dianora den korsischen Kapitän im Kampf. Rinaldini wird ebenfalls dem Liparischen Statthalter vorgeführt, dabei aber von Dianora getrennt, und nach Ragusa geschickt, wo er den Alten von Fronteja treffen soll. Dieser Alte, so stellt sich heraus, heißt eigentlich Prinz Nikanor, und zeugte mit der Römerin Laura den unehelichen Sohn Rinaldo. Der überraschend

\footnotetext{
71 [Brückner] (Anm. 45), 282.

72 Ebd., 10.

73 Ebd., 284.

$74 \mathrm{Zu}$ dieser Variante dessen, was um 1800 ein Bildungsroman sein kann, siehe Georg Stanitzek, »Arztbildungsromane. Über >bildungsbürgerlichen< Umgang mit Literatur zu Beginn des 19. Jahrhunderts«, Internationales Archiv für Sozialgeschichte der Literatur 16/1 (1991), 32-56, hier: 34.

$75 \mathrm{Zu}$ diesem, dem Konzept der bürgerlichen Familie inhärenten Imperativ, die aus den frühkindlichen Erlebnissen eingeschleiften Prägungen zu ergründen, um dadurch »an sich >selbst< kontinuierliche $>$ Entwicklungen $<$ und >Ursprünge < [zu] entdecken «, siehe Friedrich Kittler, Dichter, Mutter, Kind, München 1991, 11.

76 In einem Brief an seinen Freund Karl Gottfried Theodor Winkler vom 6. Dezember 1823 berichtet Vulpius etwa ausführlich von den zahlreichen »Bearbeitungen«, »Nachdrucken « und »Übersetzungen« seines Werks, siehe Christian August Vulpius, Eine Korrespondenz zur Kulturgeschichte der Goethezeit, hrsg. Andreas Meier, Bd. 1, Berlin, New York 2003, 362.
} 
wiedergefundene Vater ist zudem, ganz wie bei Brückner, der Pascha von Zypern. Nach einigen Verirrungen trifft Rinaldo schließlich wieder mit Dianora und dem gemeinsamen Sohn zusammen, stirbt allerdings am Ende, ebenso wie seine Geliebte, bei der Verteidigung des väterlichen Schlosses: Rinaldini fällt »auf dem Schlachtfelde «, mit »ehrenvollen Wunden bedeckt. $\ll^{77}$ Auch hier erfüllt sich das Schicksal des Räuberhauptmanns also darin, seinen Vater wiederzufinden und durch ihn zum Kriegshelden zu werden - wenngleich es, anders als bei Brückner, kein Happy End für ihn gibt.

In der Forschung ist die Fortführung, die Vulpius in Ferrandino für die RinaldiniGeschichte wählte, auf Irritationen gestoßen: Der Protagonist weise hier Eigenschaften auf, »die der Rinaldo-Figur, wie sie in der Erstausgabe entwickelt worden war, im Grunde widersprechen $\ll{ }^{78}$ Auch die überraschende Enthüllung, dass der Alte von Fronteja eigentlich Rinaldos Vater ist, war in den ersten drei Teilen keineswegs angelegt. Er gibt sich vielmehr am Ende des dritten Teils, wenn er Rinaldo niedersticht, als der Eremit und einstige Lehrer Onorio zu erkennen. ${ }^{79}$ Diese »Unstimmigkeiten « zwischen den ersten drei Teilen und der Ferrandino-Fassung, die Vulpius später durch Überarbeitungen teilweise wieder reduzierte, wurden vor allem damit begründet, dass Vulpius »sehr schnell eine Fortsetzung der erfolgreichen Räubergeschichte liefern wollte $\ll{ }^{80}$ Diese Motivation trifft sicher zu, reicht aber nicht aus, um die Diskrepanzen zwischen den ersten drei Teilen und der Fortsetzung zu erklären. Zieht man hingegen die beachtlichen Parallelen zwischen Brückners Dianora und Vulpius' Ferrandino in Betracht, dann zeigt sich, dass mehrere Elemente - darunter insbesondere die Einführung einer Vaterfigur, die zugleich der Pascha von Zypern ist - offenbar schlicht aus der Fanfiction übernommen wurden.

Wie oft bei populären Fortsetzungsromanen besteht auch hier kein streng hierarchisches Verhältnis zwischen dem >einen< Original und seinen sekundären Nachahmungen, Bearbeitungen oder Fortsetzungen, sondern ein Wechselverhältnis, das sich erst durch einen umfassenden Blick auf die vielfältigen hypertextuellen Verknüpfungen zwischen den Texten erschließt. ${ }^{81}$ Die Feststellung, dass Vulpius »Texte aus Texten « produzierte, ${ }^{82}$ trifft eben nicht nur auf prominente Vorbilder wie Schillers Räuber oder Zschokkes Abbaellino zu, sondern auch auf die Nachahmungen

\footnotetext{
77 [Christian August Vulpius], Ferrandino. Fortsetzung der Geschichte des Räuberhauptmanns Rinaldini von dem Verfasser desselben, Siebentes bis neuntes Buch, Leipzig 1801, 99.

78 Simanowski (Anm. 10), 342. Dass Rinaldini, wie er in den ersten drei Teilen gezeichnet wird, gerade kein Held sein will, sondern dass dies eher den Wünschen seines Umfelds entspricht, betont auch Nicolas Potysch, »>Held der Erzählungen<. Heroisierung, Ambivalenz und Ambiguität in Vulpius' >Rinaldo Rinaldini< (1799)«, helden. heroes. héros. E-Journal zu Kulturen des Heroischen 6 (2019), 79-88.

79 »Dein Freund Onorio konnte seine unglücklichen Lehren nur mit deinem Tode besiegeln. « [Vulpius], Rinaldo Rinaldini, Dritter Theil (Anm. 40), 227.

80 Simanowski (Anm. 10), 342.

81 Dass sich zwischen Hypo- und Hypertext Effekte der Rückkopplung und der gegenseitigen Beeinflussung ergeben können, zeigt auch Gérard Genette am Beispiel der fremden Weiterdichtungen der Romane Marivaux'. Dennoch beruht Genettes Terminologie tendenziell auf einer Hierarchie zwischen dem Original und dessen sekundären Adaptionen. Vgl. Gérard Genette, Palimpseste. Die Literatur auf zweiter Stufe, aus dem Französischen von Wolfram Bayer und Dieter Hornig, Frankfurt a. M. 1993, 14f., 222 ff.
}

82 Simanowski (Anm. 10), 213. 
und Adaptionen seiner eigenen Werke. Fanfiction, so muss mit Blick auf Vulpius' Räuberroman konstatiert werden, ist nicht zwangsläufig ein sekundäres Phänomen, sondern kann ihrerseits zur Vorlage werden.

Trotz der auffälligen Anlehnung an Brückners Dianora ist Vulpius dem Strukturprinzip seiner Geschichte allerdings in einem Punkt treu geblieben: Rinaldo Rinaldini wird bei ihm nicht zur romantischen Liebesgeschichte, die im familiären Glück ihren Abschluss findet. Vielmehr setzt sich die Irrfahrt des Räuberhauptmanns in den diversen Folgebänden fort. Obwohl die Zusammenkunft mit Dianora und dem gemeinsamen Sohn stets am Ende der Fortsetzungen steht, kommt es zwischenzeitlich immer wieder zu neuen Liebesabenteuern mit Fiametta, Fortunata oder Margalisa, wiederholten Gefechten und Fluchten. Der Umstand, dass alle Fassungen des Romans ausnahmslos mit dem Tod des Protagonisten enden, lässt darauf schließen, dass für Vulpius offenbar kein endgültiger harmonischer Abschluss der Geschichte denkbar war. Rinaldinis Grundkonflikt lässt sich weder durch die Liebe noch durch den wiedergefundenen Vater aufheben. Die schicksalhafte Struktur, die Brückner der Geschichte verleiht, wird von Vulpius zwar zunächst übernommen, im weiteren Verlauf der Handlung aber immer wieder aufgebrochen und infrage gestellt.

Es überrascht deshalb nicht, dass der Roman auch dann, als Vulpius mit der Geschichte endgültig abgeschlossen hatte, noch Fortsetzungen von fremder Hand erfahren hat. Der Roman erfreute sich noch Jahrzehnte nach seinem ersten Erscheinen ungebrochener Popularität: »Außer einigen Schiller'schen Trauerspielen«, so befand ein Rezensent des Literatur-Blatts angesichts des Erscheinens neuer Fanfiction im Jahr 1830, »ist sicher kein poetisches Werk der Neuern so oft gelesen worden als Rinaldini. ${ }^{83}$ Bezeichnenderweise teilen die Fortschreibungen des Romans tendenziell Brückners Versuch, die unendliche Wiederholungsstruktur des RinaldiniRomans stillzustellen, indem sie der Handlung eine schicksalhafte Logik unterlegen - so etwa, wenn die Geschichte von Rinaldos Sohn erzählt wird, der lernen muss, seine Affekte zu beherrschen, um nicht so zu enden wie sein Vater, und gerade dadurch dessen Schicksal als Befreier Korsikas erfüllt. ${ }^{84}$ Auch in diesem Fall geht es darum, die losen Enden des Romans weiterzuspinnen und die kontingenten Ereignisketten auf ein Ziel hinzuführen.

Doch auch wenn der Versuch, die Geschichte zu einem schlüssigen, befriedigenden Ende zu bringen oder ihre Rätsel gänzlich zu enthüllen, innerhalb der jeweiligen

\footnotetext{
83 Anonym, »Rez. Ninanor, der Alte von Fronteja, Fortsetzung der Geschichte des Rinaldo Rinaldini «, in: Literatur-Blatt, Nr. 28, 12.03.1830, 112.

84 Der Roman Nikanor, der Alte von Fronteja. Fortsetzung der Geschichte des Rinaldo Rinaldini (1828) greift anhand der Geschichte von Lionardo, Rinaldinis Sohn, noch einmal dessen Problematik auf, um sie in der Gattung des Erziehungsromans zu beheben. Damit Lionardo nicht dieselben Fehler begeht wie sein Vater, muss er lernen, seine Affekte zu beherrschen. Dies wird im Roman durch eine doppelte Sublimierung erreicht: Erstens muss Lionardo, der sich in Neapel in Doralice, die Tochter des Marchese Reali, verliebt, seine erotischen Triebe zurückhalten. Statt sich unmittelbar in das Liebesabenteuer zu stürzen und seinem »ungestühmen Sehnen« nachzugeben, muss er sich zunächst »als Mann« beweisen und sich um die auserwählte Frau verdient machen. Zweitens lernt Lionardo, der zu Beginn des Romans noch geneigt ist, »jedem Störenfriede eine Kugel durch den Leib « zu jagen, seine kämpferischen Impulse zu regulieren, indem er sie auf ein höheres Ziel - die Befreiung Korsikas - lenkt. Dabei tötet er, ohne es zu wissen, den Erzfeind seines Vaters. Moritz Richter, Nikanor der Alte von Fronteja. Fortsetzung der Geschichte des Rinaldo Rinaldini, Leipzig 1828, 156, 159, 99.
} 
Romane funktionieren mag - in der zeitgenössischen Publikationspraxis geht dieses Unternehmen nicht auf. Ebenso wie Brückners Erfindung einer Vaterfigur von Vulpius übernommen und dann für neue, abenteuerliche Verwicklungen der RinaldiniGeschichte nutzbar gemacht wurde, stellen auch die anderen Fortschreibungen kaum je einen endgültigen Abschluss dar. Tendenziell lässt sich vielmehr beobachten, dass die Aneignungen des Rinaldini-Romans zunehmend aufeinander Bezug nehmen und Motive anderer Fortsetzungen oder Adaptionen aufgreifen. ${ }^{85}$

In der Welt der Rinaldini-Geschichten gibt es bald nicht mehr bloß das eine Original, das eine Reihe von Nachahmungen hervorbringt, sondern es existiert ein gestuftes Referenzgefüge, in dem Elemente aus unterschiedlichen Vorlagen, die mitunter auch wechselseitig aufeinander Bezug nehmen, übernommen und weitergeführt werden. So veröffentlichte Heinrich August Kerndörffer etwa 1801 als »Seitenstück« zum Rinaldo Rinaldini den Roman Lorenzo oder der kluge Mann im Walde, der später überarbeitet und unter dem Titel Dianora oder die Verschwörung vom schwarzen Bunde erneut publiziert wurde. ${ }^{86}$ Kerndörffer greift die bei Vulpius angelegte Idee eines schwarzen Geheimbundes auf und macht Dianora selbst zur Banditentochter. Ihr Vater ist der Räuberhauptmann Odoardo, der wiederum eine Vorlage in Johann Jakob Brückners Roman Angelika, Tochter des großen Banditen Odoardo hat, der seinerseits ein »Seitenstück zu Schillers Geisterseher « bildet. ${ }^{87}$ Die Untersuchung von Fanfiction bietet die Chance, diese netzwerkförmige Interaktion von Texten, die gerade für den Bereich der sogenannten Genre- oder Unterhaltungsliteratur konstitutiv ist, stärker in den Blick zu nehmen. ${ }^{88}$ Die Räubereien von Rinaldini, seinem Verfasser und dessen Nachfolgerinnen und Nachfolgern nehmen kein Ende.

Funding Open Access funding enabled and organized by Projekt DEAL.

Open Access Dieser Artikel wird unter der Creative Commons Namensnennung 4.0 International Lizenz veröffentlicht, welche die Nutzung, Vervielfältigung, Bearbeitung, Verbreitung und Wiedergabe in jeglichem Medium und Format erlaubt, sofern Sie den/die ursprünglichen Autor(en) und die Quelle ordnungsgemäß nennen, einen Link zur Creative Commons Lizenz beifügen und angeben, ob Änderungen vorgenommen wurden.

Die in diesem Artikel enthaltenen Bilder und sonstiges Drittmaterial unterliegen ebenfalls der genannten Creative Commons Lizenz, sofern sich aus der Abbildungslegende nichts anderes ergibt. Sofern das betreffende Material nicht unter der genannten Creative Commons Lizenz steht und die betreffende Handlung nicht nach gesetzlichen Vorschriften erlaubt ist, ist für die oben aufgeführten Weiterverwendungen des Materials die Einwilligung des jeweiligen Rechteinhabers einzuholen.

Weitere Details zur Lizenz entnehmen Sie bitte der Lizenzinformation auf http://creativecommons.org/ licenses/by/4.0/deed.de.

\footnotetext{
85 Mit diesem Phänomen der Verknüpfung unterschiedlicher Texte bei der Fabrikation von Fanfiction befasst sich - mit Blick auf die Fankultur des 20. Jahrhunderts - Jenkins (Anm. 20), $37 \mathrm{ff}$. Siehe auch Busse (Anm. 26).

86 Heinrich August Kerndörffer, Lorenzo, der kluge Mann im Walde. Ein Seitenstück zu Rinaldo Rinaldini, Leipzig $1801 \mathrm{ff}$; ders., Dianora oder die Verschwörung vom schwarzen Bunde, Leipzig 1821.

87 Johann Jakob Brückner, Angelika, Tochter des großen Banditen Odoardo, Ein Seitenstück zu Schillers Geisterseher, Leipzig 1801.

88 Ein weiteres Beispiel für diese Interaktion bietet Johanna Isabella Eleonore von Wallenrodts literarisches »Gemählde« Karl Moor und seine Genossen beim alten Thurm (Mainz und Hamburg 1801), das sich gleichermaßen als »Fortsetzung« von Schillers Räubern und als »Seitenstück zum Rinaldo Rinaldini« präsentiert.
} 